\title{
Improved estimates of mineral trapping capacities at reservoir scale
}

\author{
A. MISHRA $^{1 *}$, R.R. HAESE ${ }^{2}$
}

${ }^{1}$ School of Earth Science and Peter Cook Center for CCS

Research, The University of Melbourne, Melbourne 3051, Australia (* correspondance:

achyutm@student.unimelb.edu.au)

${ }^{2}$ School of Earth Science and Peter Cook Center for CCS

Research, The University of Melbourne, Melbourne 3051, Australia (ralf.haese@unimelb.edu.au)

Carbon Capture and Storage (CCS) is considered to be an effective, economical and safe solution for reducing anthropogenic $\mathrm{CO}_{2}$ emmissions and becomes increasingly deployed worldwide. $\mathrm{CO}_{2}$ is injected into the subsurface where it is immoblilized by four trapping mechanisms: structural, dissolution, capillary and mineral trapping. Mineral trapping is usually assumed to be insignificant in sedimentary rocks, yet, cm-scale intraformational baffles may have a high carbon mineral trapping capacity due to the abundance of clay minerals potentially providing cations for carbonate mineral formation. This study consists of two parts: Geochemical processes and conditions controlling carbon mineralisation in intraformational baffles were determined and carbon mineralisation was quantified for a range of baffle-forming rock types using high-resolution, 2-D reactive transport simulations. Subsequently, averaged petrophysical, flow and geochemical properties were derived for intraformational baffles using the results from fine scale simulations and implemented in a 2-D reservoir-scale model representative of shallow marine sediments dominated by channel infilling. For comparison, reactive tranpsort simulations were also run on a 2-D conventional reservoir model where $\mathrm{cm}$-scale lithological heterogeneity was not captured due to coarser grid resolution. Mineral trapping capacities were computed for both models and compared. Carbon mineral trapping can be up to 2.5 times higher over 1000 years when accounting for clay-rich intraformational baffles. However, negative carbon mineral trapping can also occur when baffles contain carbonate cements, which dissolve as a consequence of the $\mathrm{CO}_{2}$ invasion. 